\title{
Erythrocyte zinc level in patients with atopic dermatitis and its relation to SCORAD index
}

\author{
Ercan Karabacak ${ }^{1}$, Ersin Aydin², Ali Kutlu³, Omer Ozcan ${ }^{4}$, Tuba Muftuoglu ${ }^{4}$, Ali Gunes ${ }^{5}$, Bilal Dogan ${ }^{1}$, Sami Ozturk ${ }^{3}$
}

1Department of Dermatology, GATA Haydarpasa Teaching Hospital, Istanbul, Turkey

2Department of Dermatology, Kasimpasa Military Hospital, Istanbul, Turkey

${ }^{3}$ Department of Allergy and Immunology, GATA Haydarpasa Teaching Hospital, Istanbul, Turkey

${ }^{4}$ Department of Biochemistry, GATA Haydarpasa Teaching Hospital, Istanbul, Turkey

${ }^{5}$ Department of Pediatrics, Dicle University Diyarbakir, Dicle, Turkey

Adv Dermatol Allergol 2016; XXXIII (5): 349-352

DOI: $10.5114 / a d a .2016 .62841$

\begin{abstract}
Introduction: Atopic dermatitis (AD) is a chronic, pruritic inflammatory disease, characterized by a relapsing-remitting course. The pathogenesis of atopic dermatitis is not completely understood, although the disorder appears to result from the complex interaction between immune abnormalities, genetic and environmental factors. Trace elements are essential for normal functioning of the immune system.

Aim: To determine zinc levels in serum and erythrocytes of patients with AD using an atomic absorption spectrometric technique and to investigate the relationship between those levels and disease activity.

Material and methods: Sixty-seven patients and 49 controls were enrolled into the study. The disease severity of AD patients was determined according to the Scoring Atopic Dermatitis (SCORAD) index. We measured zinc levels in serum and erythrocytes by the atomic absorption spectrophotometric technique.

Results: Erythrocyte zinc levels were significantly lower in AD patients than in the control group $(p<0.001)$, whereas serum zinc levels did not differ between the groups $(p=0.148)$. In the AD patient group there was a negative correlation between the SCORAD score and erythrocyte zinc levels $(r=-0.791 ; p<0.001)$.

Conclusions: The negative relationship between disease severity and erythrocyte zinc levels might suggest an immunopathological link between AD progression and intracellular zinc metabolism.
\end{abstract}

Key words: atopic dermatitis, zinc, erythrocyte, Scoring Atopic Dermatitis.

\section{Introduction}

Atopic dermatitis (AD) is a chronic, pruritic inflammatory skin disease characterized by a relapsing-remitting course that generally manifests during early childhood. The etiology of AD is still unknown; however, hereditary, genetic, racial, and environmental factors have been identified. The cause of AD consists of various factors including immunological abnormalities, exposure to allergens, and physiological and biochemical defects of the skin barrier structure [1-3].

Intake of vitamins, minerals and elements is important for the immune system in healthy people and dietary changes could have an important role in the AD etiology. Zinc is one of the trace essential elements functioning as a cofactor for several enzymes involved in metabolism and cell growth. It is highly important for all proliferating cells as well as the immune system. Since there is no specialized zinc storage system in the human body, a sufficient daily intake of zinc is required for the proper immune function [4].

There are conflicting results in serum levels of zinc in patients with AD: some authors reported lower levels [5, 6] whereas others found no differences [7, 8]. Free zinc is predominantly inside the cells and required zinc can be provided by the plasma. The serum zinc pool represents approximately one percent of the total body content [4]. Intracellular zinc levels have been reported to be a better measurement of a mild zinc deficiency and it is considered to be a good marker for the body zinc level [9].

Address for correspondence: Ersin Aydin MD, Department of Dermatology, Kasimpasa Military Hospital, 34440 Beyoglu-Istanbul, Turkey, phone: +90 212 2387900, fax: +90 212 2387902, e-mail: drersinaydin@yahoo.com Received: 16.06 .2015 , accepted: 1.10.2015. 


\section{Aim}

In this study, to determine a relation between the zinc level and severity of $A D$, we measured the erythrocyte zinc (EZ) levels in patients with AD and the healthy control group using the atomic absorption spectrophotometer (AAS) method.

\section{Material and methods}

\section{Study population}

Sixty-seven AD patients (34 females, 33 males; 17.9 \pm 8.8 average age) diagnosed using Hanifin and Rajka's diagnostic criteria [10] in 1 year (November 2012-November 2013) were included into the study. The study was approved by the local ethical committee and all patients signed informed consent.

The severity of the disease is determined by the Atopic Sermatitis Score Index (SCORAD). Atopic dermatitis is defined as mild, moderate and severe if the SCORAD index is $<25,25-50$ and $>50$, respectively [11]. Forty-nine healthy individuals were involved as the control group. The contro group and patients were at similar age and gender distributions. The patients having additional acute/chronic diseases other than allergic diseases were not included into the study. Also the patients taking zinc, systemic corticosteroid, antibiotics and systemic anti-inflammatory drugs were not included into the study.

\section{Measurement of serum and erythrocyte zinc levels}

The fasting blood samples were taken into the vacutainer blood sampling tubes containing $\mathrm{K}_{3}$ EDTA and no additive, between 08:00 and 10:00 AM. In the blood samples taken into the tubes containing $K_{3} E D T A$, the hemoglobin levels were measured by using the Abbott Sapphire hematology analyzer with its commercial kits (Abbott Laboratories, Abbott Park, IL, USA). The blood samples taken into tubes without additives were diluted with 0,2 M nitric acid 4 times. Then, zinc measurements were made using an atomic absorption spectrophotome-

Table 1. Demographic characteristics and zinc levels of patients with atopic dermatitis (AD) and controls

\begin{tabular}{lccc}
\hline Characteristic & $\begin{array}{c}\text { Patient } \\
(N=67)\end{array}$ & $\begin{array}{c}\text { Control } \\
(N=49)\end{array}$ & $P$-value \\
\hline Gender: & 34 & 25 & NS \\
\hline Female & 33 & 24 & NS \\
\hline Male & $17.9 \pm 8.8$ & $16.7 \pm 8.4$ & NS \\
\hline Age [years] & $34.4 \pm 6.1$ & $40.4 \pm 5.3$ & $<0.001$ \\
\hline $\begin{array}{l}\text { Erythrocyte zinc levels } \\
\text { [ } \mathrm{Ng} / \mathrm{g}]\end{array}$ & & & \\
\hline Serum zinc levels $[\mu \mathrm{g} / \mathrm{g}]$ & $89.9 \pm 16.8$ & $96.1 \pm 17.6$ & 0.148 \\
\hline
\end{tabular}

Values are presented as mean $\pm S D$. NS - not significant. ter (Perkin Elmer Analyst 800, Perkin Elmer Inc. MA, USA) by the absorption spectrophotometric technique [12]. The units of EZ levels were expressed as microgram Zn/gr $\mathrm{Hb}$.

\section{Statistical analysis}

To compare erythrocyte and serum zinc levels between patient and control groups, Student $t$-test was used. Statistical analyses were conducted using SPSS ver. 15.0 (SPSS Inc., Chicago, IL, USA) and $p<0.05$ was considered statistically significant. The relationship between the SCORAD index and zinc levels in serum or erythrocyte was analyzed using the Pearson correlation coefficient.

\section{Results}

The mean ages of patient and control groups were $17.9 \pm 8.8$ and $16.7 \pm 8.4$ years, respectively. There were 34 females and 33 males in the AD group, whereas there were 25 females and 24 males in the control group. The age and sex distributions of patient and control groups were similar. The laboratory parameters and patient demographics are shown in Table 1.

In the AD patient group, EZ levels were distinctly lower than those in the control group $(34.4 \pm 6.1 \mu \mathrm{g} / \mathrm{g}$ hemoglobin and $40.4 \pm 5.3 \mu \mathrm{g} / \mathrm{g}$ hemoglobin) ( $p<0.001$ ). There was no statistical difference of serum zinc levels between AD and control groups $(89.9 \pm 16.8$ and $96.1 \pm 17.6 \mu \mathrm{g} / \mathrm{dl}$, respectively; $p=0.148$ ). In AD patients there was a negative and strong statistical correlation between the SCORAD index and the EZ levels ( $p<0.001$ ) (Figure 1), but there was no statistical correlation between the SCORAD index and serum zinc levels.

When patients were grouped according to the disease severity by using the SCORAD index, the lowest EZ levels were found in the severe AD group (Table 2). In addition, the lower EZ levels in the patient group were not related to the age, gender, total Ig E levels and disease duration.

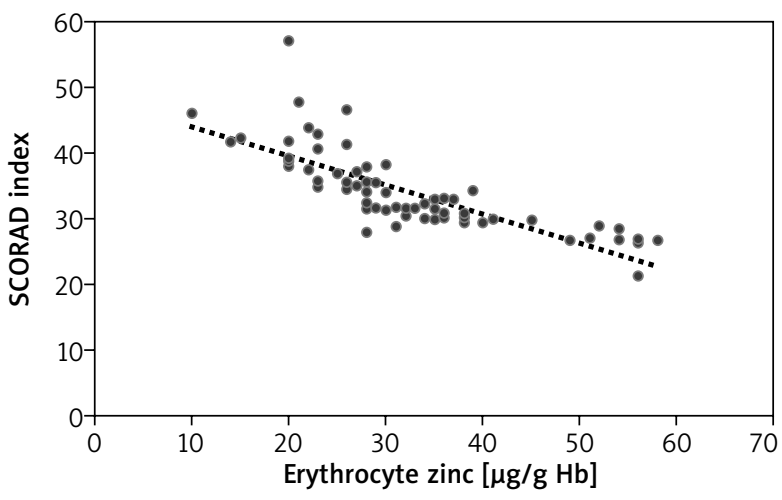

Figure 1 . The negative and statistically significant relationship between erythrocyte zinc and SCORAD index $(r=-0.791, p<0.001)$ 


\section{Discussion}

In the present study, our results showed a negative correlation of EZ levels and severity of AD and according to our knowledge, this is the first such study in the literature. Until now, a few controlled studies about the serum levels of zinc in $\mathrm{AD}$ have revealed contradictory results; some authors reported that it is lower [5, 6], whereas others demonstrated no differences [7, 8]. The same controversial result applies to the serum $\mathrm{Zn}$ level in other skin diseases like psoriasis and vitiligo [13, 14].

Zinc has been known to be an essential trace element, having a regulatory role in the immune system for a long time. It is involved in about 400 enzymes and over 2000 proteins [15]. The immune system cells have a high rate of proliferation, apoptosis and differentiation and zinc plays a major role in all these processes [4]. T cell activation as well as differentiation of $\mathrm{T}$ helper (Th) cells into their different subgroups (Th1, Th2, Th17, regulatory T cells (Treg)) are quite influenced by zinc homeostasis.

An acute zinc deficiency disorder is known with dermatitis around the limbs and body orifices, diarrhea, and impaired immunity, on the other hand, a chronic zinc deficiency can lead to the liver or kidney failure [4]. Even though the relationship between the role of zinc and AD is not obvious, experimental animal models show that a zinc-deficient diet induced AD-like eruptions and deterioration of the skin barrier function [16]. A mild zinc deficiency is seen commonly with age and this leads to a dysregulation mainly in the adaptive immunity resulting in an increased production of pro-inflammatory cytokines and shift of the Th cell balance towards Th2-response [4]. As a result, increased IL-4 production causes an enhanced unspecific B cell activation and IgE antibody production by plasma cells. This may lead to harmful immune responses such as allergic diseases via inducing a predominance of the Th2 response. Zinc homeostasis has also been demonstrated to affect dendritic cells, particularly the involvement of zinc transporter proteins during lipopolysaccharide induced upregulation of major histocompatibility complex proteins and co-stimulatory molecules [17].

Zinc is also an important antioxidant element which is an essential component of many antioxidants [18]. It has been shown that increased oxidative stress (OxS) plays a role in the pathophysiology of childhood $A D$ and the use of an antioxidant agent might be a useful strategy for the treatment of AD [19]. A low zinc level could cause the membrane barriers' problem and this problem could increase transepidermal water loss (TEWL), which makes the skin dry clinically, and the penetration of allergens could be easier [20]. This can affect the severity of the disease.

The serum concentration of zinc has been the most commonly used indicator of the zinc status. However, being influenced by a variety of factors, such as infection, stress, hormonal changes, low albumin levels, and
Table 2. Type of zinc levels and disease severity

\begin{tabular}{lccc}
\hline Type of zinc levels/ & \multicolumn{3}{c}{ SCORAD } \\
\cline { 2 - 4 } content assessed & Mild & Moderate & Severe \\
& $0-25$ & $25-50$ & $>50$ \\
& $N=16$ & $N=67$ & $N=8$ \\
\hline Serum zinc $[\mu \mathrm{g} / \mathrm{g}]$ & $95.1(16.0)$ & $89.9(16.8)$ & $90.4(20.6)$ \\
\hline Erythrocyte zinc $[\mu \mathrm{g} / \mathrm{g}]$ & $41.8(5.3)$ & $34.4(6.1)$ & $26.5(2.3)$ \\
\hline
\end{tabular}

growth rate, its diagnostic value is limited [20]. It has also been found that $99 \%$ of this trace element is being located intracellularly [21]. Therefore, the intracellular zinc level measurement has been reported to be a better method in zinc deficiency [9] and it can also reveal a borderline zinc level efficiently. The EZ level is considered the most sensitive clinical marker of the body zinc level [9]. Taking this into consideration we assessed the EZ levels with a more specific method in our study. As far as we know, there is only one study about the EZ levels in AD in the literature. Recently, Toyran et al. has found that EZ levels were lower in patients with $A D$, although they have not found a relationship between the SCORAD index and zinc levels [22]. Our study also demonstrated that the EZ contents in patients were significantly lower than in control groups. In addition, there was a negative correlation of erythrocyte zinc levels and severity of AD. The possible explanation of a statistically significant relation between EZ levels and SCORAD index, contrary to Toyran et al., may be our more sensitive zinc determination technique (AAS) than their photometric method. We used a more sensitive method which is called atomic absorption technique measuring the zinc level which can offer more reliable results. The intracellular zinc concentration is maintained by highly specialized and complex systems. The intracellular zinc level is tightly controlled by zinc transporters and zinc binding molecules for such important physiologic functions. Zinc transporters (ZnT) are localized in plasma and on intracellular membranes [23]. The defects of the membrane structure and/or this kind of protein families may be thought to be the reason of low zinc levels inside the cell. If there is a problem in the transport of zinc elements into the cell, low cellular zinc levels could be found even if it is normal in the serum. Related to this problem, the researches about the cell membrane transport protein functions and levels could help understand AD etiopathogenesis and moreover this could facilitate finding and improving specific treatment methods. Intracellular zinc homeostasis is sensitive to pathophysiological environmental changes like inflammation, OxS and heavy metals. Therefore, an increased demand or redistribution of zinc in response to inflammation may be the possible etiology of low EZ levels in AD patients in this study.

Supplementation studies with zinc showed normalized zinc levels and restoration of important functions of 
the immune system. It seems that normalized zinc levels lead to balance inversion between cell-mediated (Th1) and humoral (Th2) immunity in favor of the Th1 response. Furthermore, a recent study investigating the efficiency of oral zinc supplementation in AD demonstrated favorable effects on $A D$ supporting the abovementioned study results [20].

\section{Conclusions}

Both zinc deficiency and AD share many common immunopathological features and it is not certain to determine a cause and effect relation. The evaluation of the zinc deficiency by EZ levels determined by AAS technique instead of serum zinc concentrations in patients with $A D$ could be more informative and reliable. In addition, the negative relationship between disease severity and EZ levels might suggest an immunopathological link between AD progression and intracellular zinc metabolism. Further studies are needed to determine the effect of zinc homeostasis in AD and its severity.

\section{Conflict of interest}

The authors declare no conflict of interest.

\section{References}

1. Boguniewicz M, Schmid-Grendelmeier P, Leung DY. Atopic dermatitis. J Allergy Clin Immunol 2006; 118: 40-3.

2. Nedoszytko B, Sokołowska-Wojdyło M, Ruckemann-Dziurdzińska K, et al. Chemokines and cytokines network in the pathogenesis of the inflammatory skin diseases: atopic dermatitis, psoriasis and skin mastocytosis. Postep Derm Alergol 2014; 31: 84-91.

3. Rutkowski K, Sowa P, Rutkowska-Talipska J, et al. Allergic diseases: the price of civilisational progress. Postep Derm Alergol 2014; 31: 77-83.

4. Maywald M, Rink L. Zinc homeostasis and immunosenescence. J Trace Elem Med Biol 2015; 29: 24-30.

5. David TJ, Wells FE, Sharpe TC, et al. Low serum zinc in children with atopic eczema. Br I Dermatol 1984; 111: 597-601.

6. el-Kholy MS, Gas Allah MA, el-Shimi S, et al. Zinc and copper status in children with bronchial asthma and atopic dermatitis. J Egypt Public Health Assoc 1990; 65: 657-68.

7. David TJ, Wells FE, Sharpe TC, et al. Serum levels of trace metals in children with atopic eczema. Br J Dermatol 1990; 122: 485-9.

8. Ertunç V, Parlak M, Dane Ş, et al. The serum levels of some important elements and alkaline phosphatase in patients with atopic dermatitis. Turk J Med Sci 1996; 26: 593-6.

9. Thompson RP. Assessment of zinc status. Proc Nutr Soc 1991; 50: $19-28$

10. Hanifin JM, Rajka G. Diagnostic features of atopic dermatitis. Acta Derm Venereol (Stockh) 1980; 92: 44-7.

11. European Task Force on Atopic Dermatitis. Severity scoring of atopic dermatitis: the SCORAD index. Consensus Report. Dermatology 1993; 186: 23-31.

12. Smith JC Jr, Butrimovitz GP, Purdy WC. Direct measurement of zinc in plasma by atomic absorption spectroscopy. Clin Chem 1979; 25: 1487-91.
13. Ala S, Shokrzadeh M, Golpour M, et al. Zinc and copper levels in Iranian patients with psoriasis: a case control study. Biol Trace Elem Res 2013; 153: 22-7.

14. Zeng Q, Yin J, Fan F, et al. Decreased copper and zinc in sera of Chinese vitiligo patients: a meta-analysis. J Dermatol 2014; 41: 245-51.

15. Andreini C, Banci L, Bertini I, et al. Counting the zinc-proteins encoded in the human genome. J Proteome Res 2006; 5: 196-201.

16. Takahashi H, Nakazawa M, Takahashi K, et al. Effects of zinc deficient diet on development of atopic dermatitis-like eruptions in DS-Nh mice. J Dermatol Sci 2008; 50: 31-9.

17. Rink L, Haase H. Zinc homeostasis and immunity. Trends Immunol 2007; 28: 1-4.

18. Yuan Y, Niu F, Liu Y, et al. Zinc and its effects on oxidative stress in Alzheimer's disease. Neurol Sci 2014; 35: 923-8.

19. Omata N, Tsukahara H, Ito S, et al. Increased oxidative stress in childhood atopic dermatitis. Life Sci 2001; 69: 223-8.

20. Kim JE, Yoo SR, Jeong MG, et al. Hair zinc levels and the efficacy of oral zinc supplementation in patients with atopic dermatitis. Acta Derm Venereol 2014; 94: 558-62.

21. Vallee BL, Falchuk KH. The biochemical basis of zinc physiology. Physiol Rev 1993; 73: 79-118.

22. Toyran M, Kaymak M, Vezir E, et al. Trace element levels in children with atopic dermatitis. J Investig Allergol Clin Immunol 2012; 22: 341-4.

23. Maret W. Zinc biochemistry: from a single zinc enzyme to a key element of life. Adv Nutr 2013; 4: 82-91. 